\title{
Alteración de la respuesta de inmunoglobulina A sérica en lactantes con bronquitis obstructiva recidivante
}

DRES. H, GALlegos *, L. REBOlledo **, R. BURDACH *, A. CARMONA *** y R. SORENSEN ***.

Introducción, La IgA es el anticuerpo que se encuentra en mayor concentración en las secreciones externas, hecho que le confiere un papel importante en la respuesta inmune local frente a agentes iniecciosos y como barrera para el ingreso de antígenos inertes $(1,2,3,4,5,6,7)$.

Su deficiencia está relacionada con la aparición de diversas patologías. Esto deriva tanto de una mayor susceptibilidad a agentes infecciosos, como de una deficiente barrera a antígenos extrainos, bechos que ban sido discutidos en mayor detalle en otros trabajos en esta misma revista. ( 8 , $9,10,11,12,13,14)$.

El lactante tiene una gran predisposición a las infecciones respiratorias, entre las cuales se destaca, por su frecuencia, la bronquitis obstructiva recidivante, por su frecuencia. La susceptibilidad a esta enfermedad tiende a desaparecer alrededor de $\operatorname{los} 2$ años de edad $(15,16,17,18,19,20)$.

En el modelo de respuesta inmune en el aparato respiratorio que se expuso anteriomente (21) se postula que una respuesta deficiente de IgA secretada y de IgE podría condicionar una mayor susceptibilidad a infecciones respiratoria y a fenómenos de hipersensibilidad responsables del cuadro de bronquitis obstructiva recidivante. En este trabajo se investigó la respuesta de IgA secretada y serica en estos pacientes. Como controles se estudiaron lactantes normales de la misma edad, y un grupo de desnutridos, que si bien igualmente sufrían de infecciones, no tenían bronquitis obstructiva recidivante.

Material y Mítodo. Se estudió un grupo de 60 lactantes con bronquitis obstructiva recidivan-

\footnotetext{
* Servicto Broncopulmonares, Hospital M. Arriarán.

*t Laboratorio Investigacion, Hospital L. Calvo Mackenna.

*** Sección Inmunopatologia, Instituto Bacteriológico.
}

te en control en el Servicio de Broncopulmonares del Hospital Arriarán. Todos habían presentado tres o más crisis de bronquitis obstructiva antes del año de edad.

Se incluyó además un grupo de 19 lactantes con desnutrición proteico-calórica grave (más de $50 \%$ de déficit de peso corporal) del Servicio de Nutrición del Hospital Luis Calvo Mackenna, y un grupo de niños eutróficos $(22,23)$.

En todos los casos estudiados se determinaron los niveles de inmunoglobulinas $\mathrm{G}$ y $\mathrm{A}$ por el método de inmunodifusión radial (24). Los resultados se expresaron en Unidades Internacionales de acuerdo a patrones preparados por el Laboratorio de Referencia para Inmunoglobulinas de la O. M. S. en Lauzanne, Suiza.

En 51 pacientes con bronquitis obstructiva recidivante $\mathrm{y}$ en normales se obturvo además muestras de saliva, que se centrifugaron, filtraron y congelaron a $-20^{\circ} \mathrm{C}$ hasta su análisis. En estas muestras se determinó la concentración de IgA secretada (11 S) por el método de electroinmunodifusión (25) usando como referencia una $\operatorname{IgA} 11$ $S$ purificada.

Resultados. En el gráfico No 1 se muestra la respuesta de IgG ante infecciones a repetición en lactantes con bronquitis obstructiva recidivante y desnutridos. Ambos grupos elevan sus niveles sobre lo normal, no habiendo diferencias significativas entre ellos.

En el gráfico No 2 vemos la respuesta de IgA ante infecciones a repetición en los mismos lactantes. Hay una diferencia notable en los niveles de ambos grupos. Los desnutridos responden a las infecciones con niveles elevados, no así los lactantes eutróficos con bronquitis, que mantienen los niveles de IgA dentro de los límites normales 


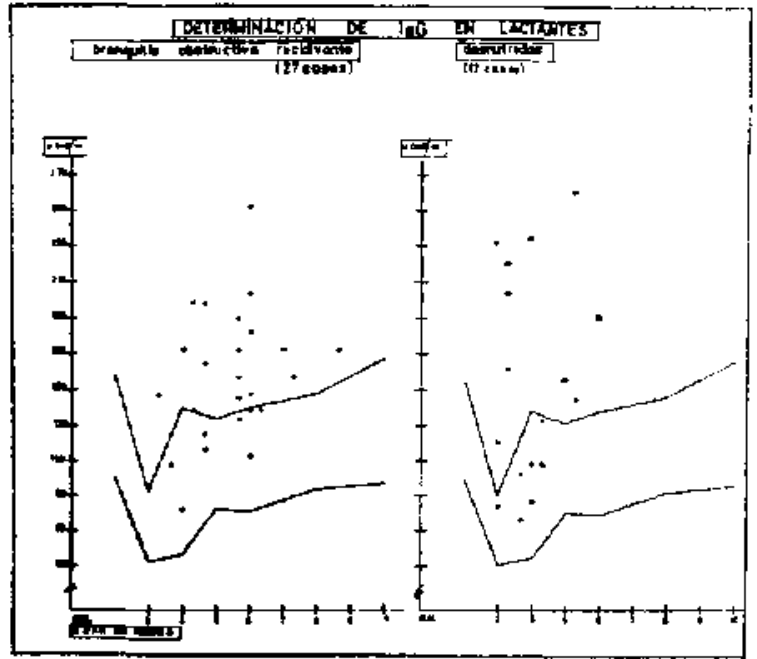

Gráfico № 1.- Respuesta de IgG en lactantes con bronquitis obstructiva recidivante frentc a infecciones a repeticion.

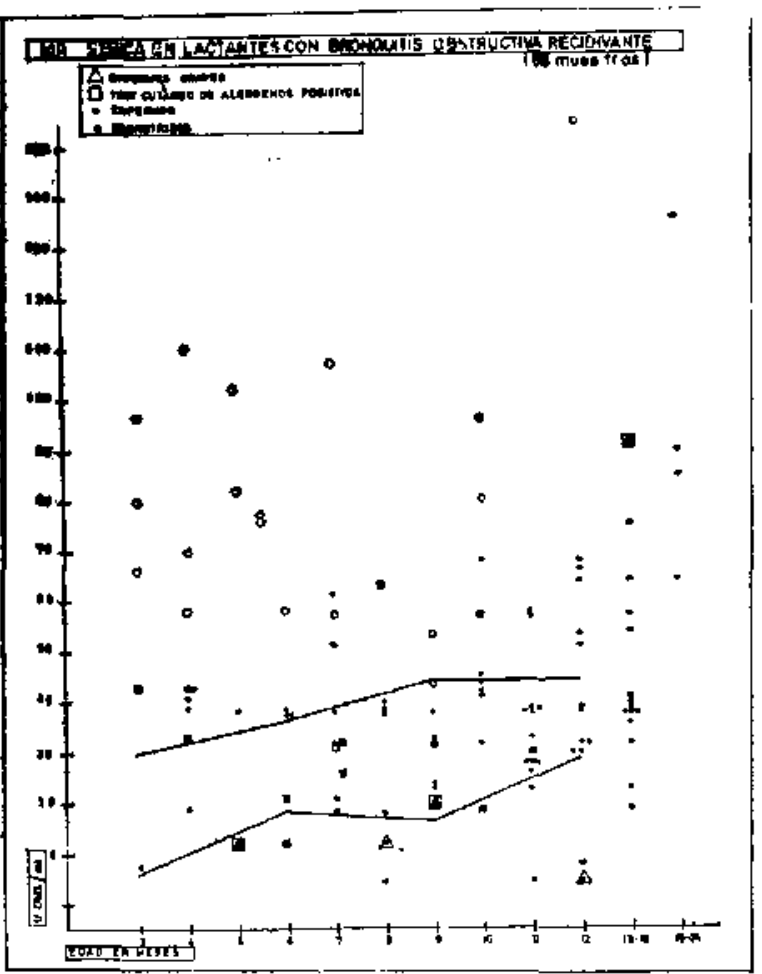

Gráfico $N^{n}$ 2.-Respuesta de IgA ante infecciones a repelicion cn el mismo grupo de Iactatites.

a pesar de sus infecciones respiratorias. Más aún, dentro del grupo que presenta niveles más bajos (11 de 28 muestras) se encuentran tres de los catalogados como graves (una o más hospitalizaciones por cuadro bronquial obstructivo).

Los resultados de la determinación de IgA en saliva se resumen en el gráfico No 3 . Se observa

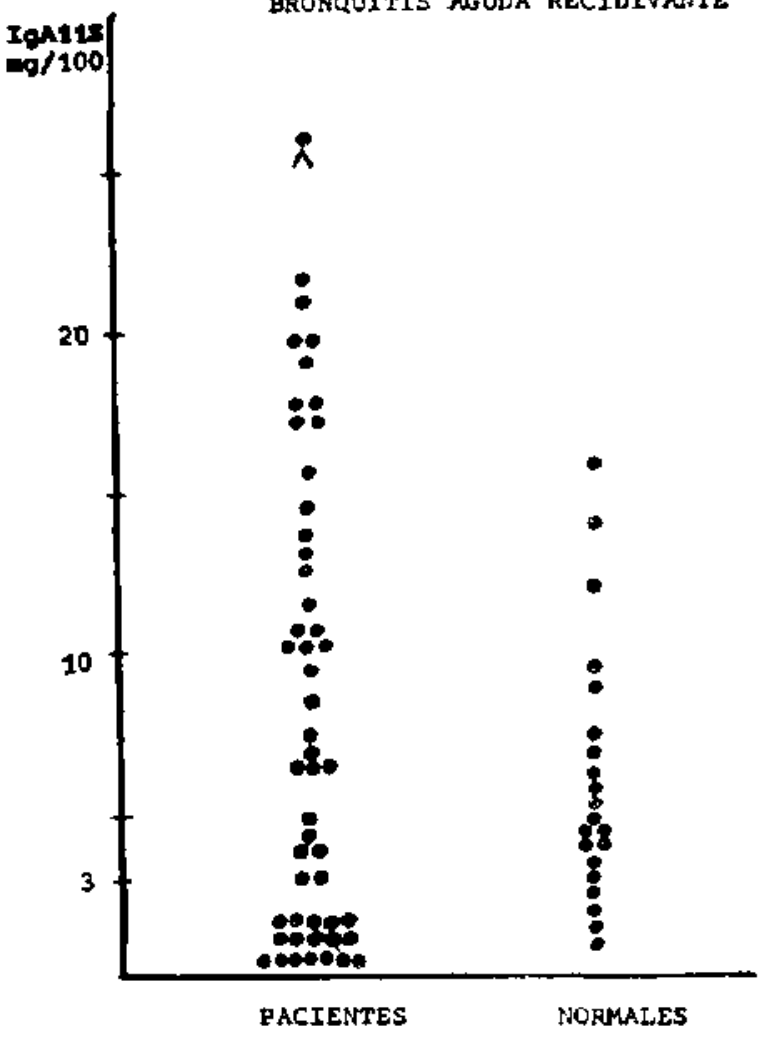

Gráfiço No 3,- Detcrminación de IgA $11 \mathrm{~S}$ en lactantes con B. O. R. y c n normales.

que 17 de 51 niños con bronquitis obstructiva recidivante presentan valores bajo $2 \mathrm{mg} \%$, que es el límite de sensibilidad de la técnica empleada, lo que corresponde a un $33 \%$. En el grupo normal igualmente se encuentran valores bajo esta cifra, pero en un porcentaje mucho menor, 3 de 23 casos $(13 \%)$.

Analizando comparativamente los niveles de IgA sérica y secretada no se ve una correlación entre ambos valores.

Discusión. El análisis de los valores de IgA e IgG sérica en pacientes con bronquitis obstructiva recidivante y en dessutridos muestran un hecho interesante: mientras frente a infecciones (respiratorias y del tracto digestivo) los desnutridos presentan una elevación de ambas inmunoglobulinas, los lactantes con bronquitis obstructiva a repetición presentan niveles de IgA normales o bajos. En estos mismos niños la IgG con frecuencia se encontró elevada.

Si se observa este hecho en conjunto con el hallazgo de valores bajos de IgA en secreciones, se puede plantear la posibilidad de una deficiente tespuesta del sistema IgA sérico y secretado, que condiciona una mayor susceptibilidad al ingreso de agentes infecciosos o substancias inertes por 
vía aérea. Esto permitiría que en este grupo de pacientes se presente con mayor frecuencia el cuadro de bronquitis obstructiva recidivante, en el cual probablemente están involucrados mecanismos de hipersensibilidad.

Los valores de IgA secretado y sérica no se relacionan en forma muy estrecha. Las deficiencias genéticamente determinadas afectan a ambas, no encontrándose $\operatorname{IgA}$ sérica ni secretada (28). En un caso de hemosiderosis pulmonar idiopática acompañada de una anemia hemolítica auto-inmune, Taboada (27) observó una disociación dc ambos sistemas, encontrando repetidamente IgA normal en el suero y disininución importante de IgA en secreciones, lo que coincidió con una ausencia de plasmocitos en las mucosas del árbol respiratorio de este paciente. En la saliva, en la cual se determinan los niveles de IgA, se observan importantes variaciones de concentración en el tiempo, lo que unido a la variación de la respuesta inmune en distintas zonas de las mucosas de un mismo individuo, lleva a que los datos presentados tengan un valor relativo. En cl futuro scria necesario tomar en cuenta estas variantes, e incluir además en el estudio respuestas específicas a determinados antígenos, seguidas en el tiempo, y analizadas comparativamente en grupos de pacientes y en normales. Por otra parte, cabe la posibilidad de que en aquellos casos en que no se encontró una disminución de IgA sćrica y/o sccretadá, cxista una alteración de la capacidad de respuesta especifica frente a determinados antígenos.

\section{RESUMEN Y CONCLUSIONES}

En lactantes afectados por bronquitis crónica recidivante se encontraron valores bajos de IgA sérica y secretada. Por contraste, los niveles de $\mathrm{IgC}$ frecuentemente estaban elevados. Igualmente $e_{n}$ desnutridos infectados. IgG e $\operatorname{Ig} A$ se encontró normal o elevada. Esto permite plantear una de. ficiente respuesta del sistema $\operatorname{Ig} A$, sérico y secretor, en pacientes con bronquitis crónica recidivante, que está siendo estudiado en más detalle en la actualidad.

\section{SUMMARY}

The immune response, as measured by the levels of IgG and IgA was determined in children with recurrent obstructive bronchitis. These levels were compared with values found in undernurrished children heavily exposed to infection, and with the values found in normal children of the same age group. IgG levels are elevated in both groups of patients over the normal values. By contrast an important difference exists in I $\subseteq A$ levels; in undernoutrished children these levels are bigh, while in the recurrent bronchitis group IgA are within the normal rar. ge or below. This could be explained by a deficient response of $\operatorname{Ig} A$ in these children, which baye a normal IgG response following the infecticus challenge. This becomes especially clear when comparin IgA and IgG levels found in the undernourrished children, in which both immunoglobulins raise following infection.

\section{REFERENCIAS}

1.- Smith R. T. Gamma A Immunoglobulin and the concept of local inmunity. Pediatrics Vol. 43: 317 , 1969.

2.-Good R. A., Rodey C. E. IgA deficiency, Antigenic Barriers and Autoimmunity. Cell. Imm. I: 147, 1970.

3.-Good R. A., Young Sung Chot. Relation to IgA and IgE to bodily defenses N. Eagl. J. Med. Vol. 284: $552,1971$.

4.-Hawosh I. C., Dilling L. Concentration of gama A globulin in serum, saliva and nasopharyngeal secreations of infants and children. J. Lab. Clin. Med. Vol. 67, N9 6: 922.

5.-Gittin D. The Differenciation and Maturación of Specific Immune Mecharisms. Act. Paed. Scand., Suppl. 172: 60, 1967.

6.- Ogra P. L., Karkazon D. T. The Role of Immunoglobulins in the mechanism of Mucosal Inmunity to Infection. Ped. Clin. North Am., Vol 17 Ne 2: $385,1970$.

7.-Bienenstack, J., Perey D. Y. E. Immune mechanisms of mucosal resistance. Med. Clin. North Am. 58, 2: 1391, 1972.

8.- Tomasi $T, B$. Immunologic Deficiency Diseases in Man. Birth Defects Series Vol. 4, 270. N. Y. Fundation, 1968 .

9.-Durmann D. 1, Roth J. Sinopulmonary recurrets infections, mental retadation and IgA-IgE deficiency. J. Ped.: 802, Nov. 1970.

10.-Schwartz D. P., Buckley R. H. Serum IgE concentration and Skin Reactivity to antiIgE antibody in IgA deficiency patiens. New Engl. J. Med., Vol. 284 № 10: $513,1971$.

11.-Fushan F. S., Zawadki. IgA Deficiency in IntersticiaI Descamative Neumonie. Am. Rev. Resp. Dis. 103: 264, 1971.

12.-Collins W. C., Lamenza C., Kokubo E. Defíciency of IgA in Serum and Respiratory Secretions. Canad. Med. Ass. J. Vol. 99: 1069, 1968. 
13.-Buckley R. H., Dees S. C. O'Fellon W. M. Serum immunoglobulins; levels in normal children and in uncomplicated cbildhood allergy. Pediatrics 41: $600,1968$.

14.-Amman, Hong R. Selective deficiency of IgA asociated to autoimmunity. J. Lab. Clin. Med. 74: 846, 1969.

15.-Grupo de estudio de Enjermedades Broncopulmonares. Enfermedades Bronquiales de la Infancia. Rev. Chilena Ped. № 3: 230, 1970.

16.-Burdach R., Frez A. Enfermedad Bronquial obstructiva Crónica en la infincia. Rev. Chil. Ped. No 2: 785, 1967.

17.-Selarder P. Asmatic Symptoms in The First Year of Life. Acta Paed. 49: 265, May, 1960.

18.-Eisen $A, H$. The Role of Infection in Allergic Disease. Ped. Clin. North Am. Vol. 16, No 1; 67, 1969.

19.-Burdach y Col. Bronquitis Obstructiva Recidivante. Congreso de Ped, Punta Arenas, Chile, 1971.

20.-Witting H. 1., Chang C. H. Bronquiolitis or Asthma? Ped. Clin. North Am. Vol. 16, No I: 55, 1969.

21.-Sörensen, $R$., Rebolledo, $L$. Las enfermedades del aparato respiratorio vistas por el inmunologo. Pu- blicaüo en este mismo número de ka Rev. Chjlena de Pediatría.

22.-Rowe $D$. S. Concentration of serum immunoglobulins in haltby adult maies expressed in International Unites er milliliter. Lancet, por publicarse.

23.-Sörensen R., Hurtado R., Siagno S., Meléndez M., Carmona A. Inmunidad Humoral en miños chilenos. Desarrollo de las Inmunoglobulinas séricas expresado en Unidades $\mathbf{O}$. M. S. Bol. of Sen. Panam. por publicarse.

24.-Mancini, C., Carbonara A., Heremans, J. Immunochemical quantitation of antigens by single radial immuno-diffusion. Immunoquemistry $2: 235$, 1965.

25.-Merrill, D., Hartley, T., and Claman, H. Electroinmuno diffusion (E. I. D.) : a simple rapid method for quantification of Igs in dilute biological flujds. J. of Lab. and Clin. Med. 69, 151, 1967.

26.- Sörensen, R.- Defíciencia de IgA. Publicado en este mismo número de la Rev. Chilena de Pediatría.

27.-Taboada, H. Comunicación personal. 